\title{
Eye gaze influences working memory for happy but not angry faces
}

\author{
Margaret C. Jackson \\ School of Psychology, University of Aberdeen
}

Running Header: Gaze and Emotion Interactions in Face WM

Correspondence to:

Margaret C Jackson

School of Psychology

William Guild Building

Kings College

University of Aberdeen

Aberdeen

AB24 3FX

Tel: 01224272236

Email: m.jackson@abdn.ac.uk

Main text $=5334$ words 


\begin{abstract}
Previous research has shown that angry and happy faces are perceived as less emotionally intense when shown with averted versus direct gaze. Other work reports that long-term memory (LTM) for angry (but not happy) faces was poorer when they were encoded with averted versus direct gaze, suggesting that threat signals are diluted when eye contact is not engaged. The current study examined whether gaze modulates working memory (WM) for angry and happy faces. In stark contrast to LTM effects, WM for angry faces was not significantly modulated by gaze direction. However, WM for happy faces was significantly enhanced when gaze was averted versus direct. These findings suggest that in WM - when rapid processing and an immediate response is required - averted gaze may alter the meaning behind a smile, and make this kind of expression particularly salient for short-term processing.
\end{abstract}

Keywords: Emotion; Gaze; Working Memory; Faces; Happy 


\section{Introduction}

During social interaction it is important to perceive and understand signals of social intent conveyed by others so that we can respond and plan our own behaviour appropriately. Two very powerful signals of intent are facial expression and direction of eye gaze. Facial expressions of emotion can provide clear insight into another person's feelings and state of mind. Where someone is looking can cue important information in the external environment, but can also convey internal feelings and intentions. Research has shown that direct eye contact can intensify the social connection and attention paid to one another during interaction (e.g., Freeth, Foulsham, \& Kingstone, 2013). In contrast, averting one's gaze from another can convey a variety of more complex and subtle social signals such as nervousness and deception (Vrij, 2002), deference, or boredom (see Kleinke, 1986). Furthermore, gaze direction is thought to communicate the intensity of our emotions, with more direct gaze maintained when communicating strong compared to weaker positive and negative feelings (Kimble \& Olszewski, 1980).

A growing number of studies show that gaze and emotional expression are not independent, and can interact in certain ways to influence person perception. One set of studies has found that emotion and eye gaze interact to influence how particular emotions are perceived and decoded. It is argued that some emotions such as anger and happiness signal an intention to approach, while other emotions such as fear and sadness signal avoidance. From this approach/avoid distinction of emotional expression, a "shared signal hypothesis" has been proposed in which emotions are perceived to be more intense when gaze direction matches the expected intention (approach or avoid) linked to the expression (Adams \& Kleck, 2003, 2005). In illustration of this, Adams and Kleck (2003) found that categorisation of angry faces as angry was slower when faces were paired with averted than direct gaze, while categorisation of fearful faces as fearful showed the opposite effect in that responses were faster when fearful gaze was averted. Advancing on this, Adams and Kleck (2005; Experiment 3) found that approach emotions (anger and happiness) were perceived as more intense when paired with direct gaze, while avoid emotions (sadness and fear) were perceived as more intense when paired with averted gaze. This effect was replicated for angry and fearful faces (Sander, Grandjean, Kaiser, Wehrle, \& Scherer, 2007; but see Bindemann, Burton, \& Langton, 2008 for contrasting evidence).

There are two alternative hypotheses to the "shared signal" account regarding the integration of emotion and gaze information, the "appraisal hypothesis" and the "direct gaze hypothesis" (see Graham \& LaBar, 2012 for an overview). The "appraisal hypothesis" 
(Sander et al., 2007) considers the behavioural relevance of specific emotion-gaze combinations in terms of both the signal conveyed by another individual and the consequence to the observer. For example, an angry face with direct gaze is argued to be more behaviourally relevant to the observer than an angry face with averted gaze, as the direct threat signal implies an attack. On the other hand, a fearful face is more appropriately paired with averted gaze than with direct gaze to signal indirect threat to the observer from something or someone in the surrounding environment. The "direct gaze hypothesis" derives from the theoretical perspective that attention directed towards an observer via direct eye contact enhances the processing of other facial signals and attributes such as expression and attractiveness, due to eye contact increasing the self-relevance of the interaction (see Graham \& LaBar, 2012).

The research outlined above has focussed on how expression and gaze combine to influence how we perceive others. However, social interactions unfold over time to advance beyond the initial percept and engage short-term, working memory (WM) and long-term memory (LTM) for person information. WM and LTM are traditionally considered to be distinct (e.g., Atkinson \& Shiffrin, 1968) and used for different purposes, and there are some key differences between them. WM is an 'online' system that operates within the timescale of a few seconds (this can be extended slightly if active rehearsal is enabled). It is fundamental for temporary storage, manipulation and updating of information in order to facilitate the immediate planning and execution of future thoughts and behaviours. It can be considered as a window of thought that exists up to 30 seconds in the past and up to 30 seconds in the future. WM is engaged when information becomes temporarily unavailable for perceptual processing, which in vision can occur frequently during eye, head and body movements. WM is severely limited in capacity (for example, only two faces can be retained successfully in WM at any one time; Jackson \& Raymond, 2008) so cognitive resources have to be allocated efficiently and appropriately. WM is fundamental during social interaction, allowing us to not only keep track of our social partners (identity memory), but to monitor fleeting changes in facial characteristics, such emotional expression and gaze direction, so that these social cues can be detected and utilised effectively at that moment in time. In contrast, LTM operates in a timescale of a few minutes up to years and is unlimited in capacity. While WM is used for immediate goal-directed thought and behaviour, person information in LTM can be stored and retrieved for later use if and when required. In terms of learning, it is proposed that WM acts as a gate through which information travels before it is stored in LTM (Nikolić \& Singer, 2007). 
There is relatively extensive literature on how gaze and facial expression separately influence WM and LTM for face identity, but research into how gaze and emotional expression interact to influence memory is severely lacking. A study using only neutrally expressive faces showed that LTM for faces was better when they were encoded with direct than averted gaze (Mason, Hood, \& Macrae, 2004). Mason and colleagues proposed that direct gaze enhances and elaborates face encoding which in turn facilitates later recognition (in line with the "direct gaze hypothesis"). A large body of research using only direct gaze faces shows that emotional expression influences how accurately person identity information is recalled from LTM and WM. In LTM, there is a recall advantage for happy faces overall (e.g., D'Argembeau and Van der Linden, 2007; Liu, Chen, \& Ward, 2014; Shimamura, Ross, \& Bennett, 2006). In WM, however, a threat benefit is found. Using a simple delayed discrimination task where participants were required to state whether a test face was present or not a second or so earlier, WM was significantly enhanced when the faces encoded into WM conveyed an angry versus happy or neutral expression (Jackson, Linden, \& Raymond, 2014; Jackson, Wolf, Johnston, Raymond, \& Linden, 2008; Jackson, Wu, Linden, \& Raymond, 2009; Thomas, Jackson, \& Raymond, 2014; see also Sessa, Luria, Gotler, Jolicoeur, \& Dell'acqua, 2011, and Stiernströmer, Wolgast, \& Johansson, 2015). This angry benefit in WM is thought to reflect a survival response that triggers more detailed or elaborate encoding of threatening information in order to facilitate an appropriate, immediate response.

When it comes to the question of how expression and gaze interact to modulate memory accuracy, only one study to date has examined this in LTM. Nakashima, Langton, and Yoshikawa (2012) found that incidental memory recall for angry face identities that were initially judged for age was significantly worse when they were initially viewed with averted gaze compared to direct gaze. However, LTM for happy faces was unaffected by gaze direction. Their findings suggest that, in LTM at least, gaze aversion dilutes the threat signal conveyed by an angry expression, which may in turn reduce engagement and motivation to process person identity details other than those that are immediately task relevant. This pattern of results best aligns with the "appraisal hypothesis" of expression-gaze interactions.

The current study sought to investigate for the first time how emotional expression and gaze direction interact to influence WM for faces. Across two experiments, two angry or two happy male faces were presented for encoding into WM. After a 1000ms maintenance interval, a single neutral test face was presented for retrieval and participants made an identity 'match' or 'non-match' response. Emotion and gaze were task-irrelevant, but 
participants were explicitly tasked with memorising face identity information. Crucially, all faces on each trial showed either direct or averted gaze (50\% of trials each). In Experiment 1a gaze was randomised across trials, whereas in Experiment $1 \mathrm{~b}$ gaze was blocked. Both angry and happy expressions are considered to be approach emotions, so according to the "shared signal hypothesis" (and in accordance with studies which showed diluted perception of emotion in angry and happy faces with averted compared to direct gaze, e.g., Adams \& Kleck, 2003, 2005; Bindemann et al., 2008), one might predict that averted (avoid) gaze might impair WM for both angry and happy faces. However, Nakashima et al. (2012) showed an expression-specific effect of gaze on LTM for angry but not happy faces. Thus, if gaze and expression interact in the same way in LTM and WM, it could be predicted that WM for happy faces will be unaffected by gaze direction, while WM for angry faces will be poorer when gaze is averted versus direct. As mentioned above, the angry benefit in WM found using direct gaze faces is thought to reflect the temporary retention of more detailed or elaborate threatening information (Jackson et al., 2014). Therefore poorer WM for averted vs. direct gaze angry faces is predicted if averted gaze dilutes the level of threat perceived from an angry face, and thus reduces the degree of detail encoded into WM.

However, as outlined earlier WM is different to LTM in timescale and thus importantly in terms of the immediacy of the perceptual judgement and response required. In WM tasks, information has to be rapidly and effectively encoded for immediate recall a few seconds later, and this encoding is explicit and goal-directed for that purpose. LTM on the other hand does not necessarily require such engagement of immediate goals and information can be encoded actively or passively for later recall that is either expected or unexpected. In Nakashima et al.'s (2012) study, face identity information was not explicitly required to be encoded during the age judgement phase, and LTM for identity was tested using a surprise recall task. The active and immediate nature of WM may alter how we interpret and respond to expression-gaze combinations. If gaze aversion dilutes the intensity of happiness expressed by a smiling face (Adams \& Kleck, 2005), it is possible that such faces are rapidly processed not as a benign, more neutral social signal, but as ambiguous and therefore potentially threatening in their intent. If this were the case, happy faces with averted gaze may be more behaviourally relevant than those with direct gaze (according to the "appraisal hypothesis"), and thus better encoded into WM.

\section{Methods}

\section{Participants}


Power analysis using $\mathrm{G}^{*}$ Power (Version 3.1.9.2), using the angry versus happy effect size from Experiment $1(\mathrm{~N}=22)$ in Jackson et al. (2014), indicated that a sample of at least 14 participants was required to achieve a moderate effect. Thus we at least doubled that to increase power here. Thirty participants in Experiment 1a (15 females, 15 males; mean age 21 years), and 36 participants in Experiment $1 b$ (18 females, 18 males; mean age 21 years) were recruited from the University of Aberdeen student participant pool, some voluntarily and others in return for course credits. One female participant was removed from the analysis of Experiment $1 \mathrm{~b}$ due to floor performance, leaving a sample of 35 participants here. All had normal or corrected-to-normal vision, and were consented and debriefed according to the School of Psychology, University of Aberdeen ethics requirements.

\section{Stimuli}

Six male faces were taken from the Ekman and Friesen (1976) database, each in their angry, happy, and neutral expression (18 images in total). Original faces showed direct gaze, and each image was manipulated using Corel Paintshop Pro X5 to show averted gaze by moving and blending the pupil and iris. Each face was cropped with a uniform oval outline to remove hair and other external features, was made greyscale, and subtended approximately 2.39 by 3.34 degrees of visual angle (on-screen size: $2.5 \mathrm{~cm}$ x $3.5 \mathrm{~cm}$ ). Both experiments were conducted on a Dell Optiplex 780 with 1024 x 1280 resolution.

\section{Design and Procedure}

In Experiment 1a, gaze (direct, averted) was randomised across trials. In Experiment $1 b$, gaze was blocked and counterbalanced in order to check whether the effects of gaze on WM for emotional faces in Experiment 1 could be due to the unpredictability of gaze direction from trial to trial. All other elements of each experiment were identical. Participants were given 16 practice trials ( 8 direct gaze, 8 averted gaze) to start. The main experiment comprised 240 trials in total, 120 with direct gaze (60 angry, 60 happy; randomised) and 120 with averted gaze (60 angry, 60 happy; randomised). Participants controlled the start of each trial with a button press, to allow frequent and flexible breaks. A central fixation cross was presented for $1000 \mathrm{~ms}$, followed by the encoding array of two faces (either angry or happy) for 2000ms. Two faces were shown at encoding as prior research has shown that face WM capacity limits are around two faces (Jackson \& Raymond, 2008). Thus one face would be too easy and yield near ceiling performance while more than two faces may make the task too difficult. The two encoding faces were presented on either side of the central fixation cross 
and always shared the same expression. Participants were instructed to remember face identity, and that expression and gaze direction were task-irrelevant. Following a 1000ms blank maintenance interval with only the fixation cross visible, a single test face with neutral expression was shown in the centre of the screen. Participants stated whether the test face matched in identity or not to one of the two faces just seen at encoding (50\% match, $50 \%$ non-match trials, randomised). A response window of 3000ms was applied. An example trial is illustrated in Figure 1a and the four gaze-emotion conditions are summarised in Figure 1b.

1a

Trial structure

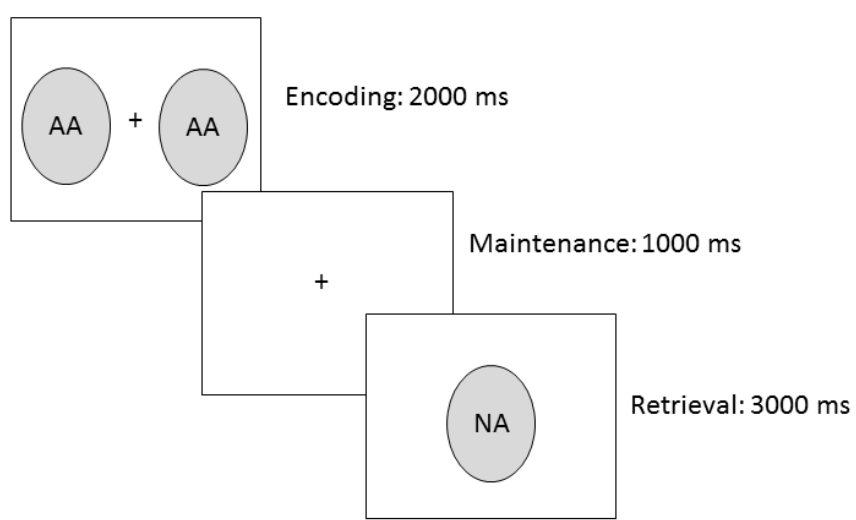

$1 b$

Encoding conditions

Averted Direct

Angry

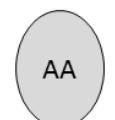

$A D$

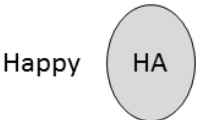

HD

Figure 1. (a) Schematic trial procedure in the WM task. Real faces were used in the actual task. Faces at encoding showed either an angry expression or happy expression with either averted or direct gaze (angry-averted faces - AA - are illustrated here). The test face was always of neutral expression and always matched the gaze direction of the encoding faces on a particular trial (i.e., NA denotes 'neutral-averted'). (b) The four Emotion-Gaze conditions: Angry-Averted (AA), Angry-Direct (AD), Happy-Averted (HA), Happy-Direct (HD).

\section{Results}

Hit rates (the proportion of correct 'yes' responses on test item present trials) and False Alarms (FA; the proportion of incorrect 'yes' responses on test item absent trials) were computed into d' scores $\left(d^{\prime}=z H i t s=z F A\right)$. This provides a more sensitive measure of memory discrimination across signal present and signal absent trials at retrieval than overall proportion (or percent) correct data, because it measures memory sensitivity while accounting for response bias (Green \& Swets, 1966). 


\section{Experiment 1: Randomised Gaze}

A repeated-measures ANOVA on percent correct data with emotion (angry, happy) and gaze (direct, averted) as within factors revealed a significant interaction between emotion and gaze $\left(F(1,29)=6.51, p=.02, \eta_{p}^{2}=.18\right.$, observed power $\left.=.69\right)$. To examine this interaction, planned (uncorrected) paired $t$-tests were computed on separate angry and happy face data to directly assess the hypothesis that gaze would modulate WM for angry but not happy faces. The results ran counter to this prediction. When faces were angry, gaze did not significantly modulate WM accuracy $(t(29)=0.65, p=.52$, Cohen's $d=.08)$. When faces were happy, WM was significantly more accurate when encoded with averted than direct gaze $(t(29)=2.31, p=.03$, Cohen's $d=.30)$, see Figure 2a. There were non-significant main effects of emotion $\left(F(1,29)=1.52, p=.23, \eta_{p}{ }^{2}=.05\right.$, observed power $\left.=.22\right)$ and gaze $(F(1$, 29) $=1.51, p=.23, \eta_{p}^{2}=.05$, observed power $=.22$ ).

Although it was not the aim of the current study to assess emotion effects in each gaze condition, to further examine the interaction additional post-hoc tests on separate averted and direct data were computed (uncorrected). When gaze was averted WM was significantly better for happy than angry faces $(t(29)=2.50, p=.02$, Cohen's $d=.29)$, however when gaze was direct there was no significant influence of emotion $(t(29)=0.72, p=.48$, Cohen's $d=$ .08 ). One might have expected an angry benefit with direct gaze (in line with previous findings of Jackson et al., 2014, and also Jackson et al., 2008, 2009), but it is possible that the presence of different gaze directions in this task changed the socio-emotional context sufficiently to alter how the emotional expressions are perceived in general. 


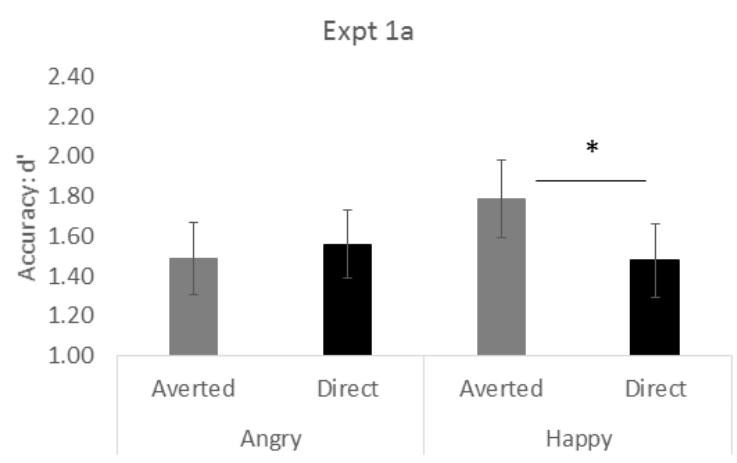

$2 b$

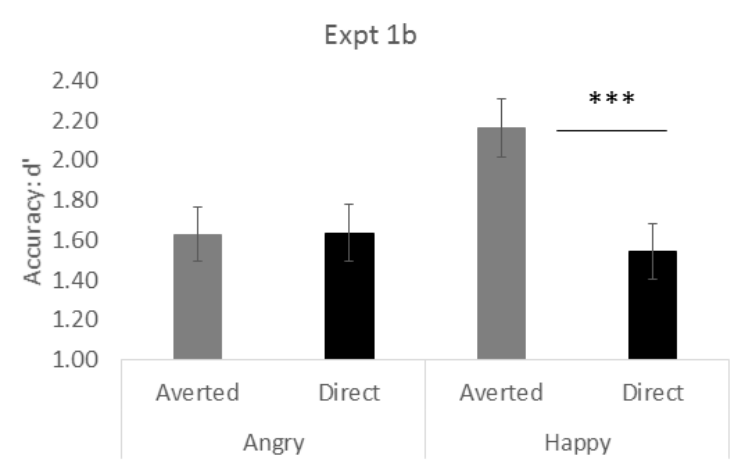

Figure 2. (a) Results from Experiment 1a, in which both emotion and gaze were randomised from trial to trial; (b) Results from Experiment 1b, in which emotion was randomised but gaze was blocked. $*=p<.05, * *=p<.01, * * *=p<.001$.

\section{Experiment 1b: Blocked Gaze}

A repeated-measures ANOVA with emotion (angry, happy) and gaze (direct, averted) as within factors revealed a significant interaction between emotion and gaze $(F(1,34)=$ $18.41, p<.001, \eta_{p}{ }^{2}=.35$, observed power $\left.=.99\right)$, replicating the pattern of effects found in Experiment 1a. When faces were angry, gaze did not significantly modulate WM accuracy $(t(34)=0.05, p=.97$, Cohen's $d=.01)$. When faces were happy, WM was significantly more accurate when encoded with averted than direct gaze $(t(34)=4.26, p<.001$, Cohen's $d=$ .73). See Figure 2b. Further post-hoc tests (uncorrected) showed significantly better WM for happy than angry faces when gaze was averted $(t(34)=5.40, p<.001$, Cohen's $d=.65)$, and no significant influence of emotion when gaze was direct $(t(34)=0.82, p=.42$, Cohen's $d=$ .11). Unlike Experiment 1a, here there was a significant main effect of emotion $(F(1,34)=$ $8.02, p=.01, \eta_{p}^{2}=.19$, observed power $\left.=.79\right)$ where WM was better for happy faces $(\mathrm{M}=$ $1.85, \mathrm{SE}=0.12)$ than angry faces $(\mathrm{M}=1.63, \mathrm{SE}=0.11)$ overall. In addition, there was a significant main effect of gaze $\left(F(1,34)=5.21, p=.03, \eta_{p}{ }^{2}=.13\right.$, observed power $\left.=.60\right)$, where faces with averted gaze $(\mathrm{M}=1.90 ; \mathrm{SE}=0.13)$ were remembered better overall than faces with direct gaze $(\mathrm{M}=1.59 ; \mathrm{SE}=0.13)$. The overall happy benefit and averted gaze benefit is likely driven by the superior combination of averted gaze with a happy expression, which further post-hoc testing showed was also significantly better than WM in the angrydirect condition $(t(34)=3.13, p=.004$ uncorrected, Cohen's $d=.62)$. 
Mixed ANOVAs with emotion and gaze as within factors and gaze block order (direct first; averted first) as a between factor, showed a non-significant three-way interaction between gaze block order, emotion, and gaze $\left(F(1,33)=3.07, p=.09, \eta_{p}{ }^{2}=.09\right.$, observed power $=.40$ ), so block order was not considered any further.

Finally, comparing across experiments $1 \mathrm{a}$ and $1 \mathrm{~b}$ showed a non-significant main effect of experiment $\left(F(1,63)=0.72, p=.40, \eta_{p}{ }^{2}=.01\right.$, observed power $\left.=.13\right)$, and nonsignificant interactions between experiment and any other condition (experiment $\mathrm{x}$ emotion: $F(1,63)=0.90, p=.35, \eta_{p}{ }^{2}=.01$, observed power $=.15$; experiment $\mathrm{x}$ gaze: $F(1,63)=1.21$, $p=.28, \eta_{p}{ }^{2}=.02$, observed power $=.19$; experiment $\mathrm{x}$ emotion $\mathrm{x}$ gaze: $F(1,63)=1.40, p=$ $.24, \eta_{p}{ }^{2}=.02$, observed power $=.22$ ). Thus, while blocking gaze appeared to have strengthened the effect of combining a happy expression with averted gaze compared to randomising gaze in Experiment 1a, there are no statistically distinguishable differences in expression-gaze interaction effects between experiments.

\section{Discussion}

To summarise, in both experiments 1a (randomised gaze) and 1b (blocked gaze) WM for happy faces was enhanced when they showed averted versus direct gaze, while WM for angry faces was not affected by gaze direction. This pattern of results does not align with the "shared signal hypothesis" of how gaze and expression interact during face perception. Both angry and happy faces are considered to signal approach, and are therefore thought to be more congruently paired with a direct gaze than averted gaze. This could be predicted to result in poorer WM for both angry and happy faces with averted than direct gaze. However, the emotion-specific effect found here indicates that angry and happy expressions are influenced differently by gaze when WM is engaged, and the opposite effect was found. The current finding also contrasts with the "direct gaze hypothesis", as direct gaze faces were remembered worse in WM than averted gaze faces overall in Experiment 1b. The "appraisal hypothesis" remains the most parsimonious interpretation of the data shown here. When WM is engaged, gaze may alter how we rapidly appraise and encode benign individuals (i.e., smiling faces), but appraisal of faces that signal malevolent intent (i.e., anger) are immune to differences in gaze - an angry person is angry regardless of where they look.

However, this pattern of results is in direct contrast with expression-gaze interaction effects found in LTM. Nakashima et al. (2012) found that LTM for happy faces was 
unaffected by gaze direction while angry faces recall was poorer when they showed averted versus direct gaze. Why is there such dissociation between emotion-gaze interaction effects in WM versus LTM? As discussed earlier, using only direct gaze faces a happy face benefit in LTM has been established (e.g., D'Argembeau and Van der Linden, 2007; Liu et al., 2014; Shimamura et al., 2006) while an angry face benefit exists in WM (Jackson et al., 2008, 2009, 2014; Thomas et al., 2014). The happy benefit in LTM is thought to exist to facilitate the creation and maintenance on prosocial affiliations over time, while the angry benefit in WM is thought to reflect an effective and immediate response to social or physical threat. It is possible that LTM representations for happy faces may be so robust that other characteristics of the face which are not task-relevant (i.e., gaze) are deprioritised over time and thus do not influence recall. The same logic could be applied to the immunity of angry faces in WM to the direction of gaze, if we consider that threat signals strengthen angry face representations in WM. Perhaps in the short-term (from second to second), the level of threat conveyed by an angry expression is stable and robust and dominates over other facial signals such as gaze.

The nature of the memory task and what information is attended to during encoding must also be considered when comparing the pattern of gaze-expression interactions in WM and LTM. In both the current study and in Nakashima et al.'s (2012) LTM task, expression and gaze information were task irrelevant and so any influence of these signals on face recognition is incidental. However, in the LTM task face identity was also task irrelevant as participants were required to judge age and a surprise memory task was administered 5 minutes later. Thus, gaze-expression interaction effects on LTM for face identity reflect incidental encoding of identity into LTM. In the WM task used here, participants were explicitly instructed to memorise face identity (in WM tasks in general encoding is directive and explicit). Furthermore, the role of WM in the LTM task used by Nakashima and colleagues is not clear. Participants made an age judgement on each face while the face was present (which would not require WM), and given that there was no instruction to memorise the faces there may have been little or no engagement of WM processes to aid learning. It would therefore be important for future research to examine the impact of gaze-expression combinations on explicit identity encoding during a LTM task - which may engage WM to a greater degree - to ascertain whether this could account for the contrasting gaze-expression interaction effects found. One might predict that explicit identity encoding and the engagement of WM in active LTM learning may alter how the faces are appraised and show the same pattern of gaze-expression interaction as found here. Note, it would be very difficult to assess implicit identity encoding during a WM task, as the mere measure of probing 
identity in the retrieval phase of a trial would alert participants to more actively encode that information on subsequent trials.

It is also possible that how attention is allocated during encoding - e.g., whether to specific facial features or to more configural (broader, global visuo-spatial) information may influence how expression and gaze combine to influence identity recognition in WM. Configural processing is considered necessary for effective face recognition (e.g., Farah, Wilson, Drain, \& Tanaka, 1995), and recent work suggests that averted gaze disrupts configural processing of faces to induce more feature-based processing (Sessa \& Dalmaso, 2016; Young, Slepian, Wilson, \& Hugenberg, 2014). In particular, Sessa and Dalmaso measured the amplitude of the sustained posterior contralateral negativity (SPCN) during a neutral faces WM task, using SPCN to indicate of the amount of information maintained in WM (e.g., Sessa et al., 2011). They found increased SPCN amplitude for neutral faces encoded with averted gaze than direct gaze, and suggest that averted gaze faces are encoded in a more detailed, featural fashion that direct gaze faces. In the current study, if increased feature-based processing of averted gaze faces occurred, this proffered a greater advantage to the encoding of happy faces than to angry faces into WM. Speculatively, this may be achieved by directing more attention to the eyes of happy faces. The eye region in general is shown to be more useful for face identity recognition (e.g., Barton, Radcliffe, Cherkasova, Edelman, \& Intriligator, 2006; Schyns, Bonnar, \& Gosselin, 2002). However, the mouth region is typically favoured over the eye region during the processing of happy faces (Eisenbarth \& Alpers, 2011; Schurgin et al., 2014). If increased featural processing encourages greater processing of the eye region of happy faces, this may have led to enhanced and more detailed encoding of identity-related information into WM for happyaverted vs. happy-direct faces ${ }^{1}$. It would be interesting for future research to measure SPCN amplitude for the different gaze-expression combinations in WM used here in order to test this prediction. In contrast, angry faces afford more attention to the eyes than the mouth (Schurgin et al., 2014), so this could account for the lack of gaze effect on angry faces in WM found here. Eyetracking may also be useful to measure the nature of attention allocation via fixation patterns on specific gaze-expression combinations, during face perception at the WM encoding stage.

\footnotetext{
${ }^{1}$ Thank you to an anonymous reviewer for this suggestion.
} 
From a broader behavioural appraisal perspective, these results suggest that in WM when rapid processing and an immediate response is required - the meaning behind a smile may be altered as a function of whether that person engages or avoids eye contact in that moment. The fact that WM is enhanced and not impaired for happy-averted versus happydirect faces is intriguing. At first this effect may appear to be in direct contrast with existing literature on emotional expression and gaze interactions, which suggests that the degree of happiness perceived from a smile is diluted when eye gaze is shifted away from the observer (Adams \& Kleck, 2005). However, reducing happy signals does not necessarily mean that the face is rendered more 'neutral'. Results from Experiment 1b showed that WM for happyaverted faces was superior to WM for angry-direct and angry-averted faces. This suggests that happy faces with averted gaze are highly salient in this task and confer a particularly strong benefit on encoding face identity information into WM. Thus happy-averted faces are unlikely to be more neutral in nature than happy-direct faces, at least in this task context. What might make them so salient?

If there is diluted perception of happiness when eyes are averted (Adams \& Kleck, 2005), then what social intent or message might this particular expression-gaze combination convey? Evil villains in cartoons and films are sometimes caricatured to depict a smile with averted eyes, to show that they are plotting something that is exciting to them but harmful to others. It is possible, therefore, that a smiling face which does not engage eye contact may be perceived as somewhat suspicious, sly, and to have malevolent thoughts, to be threatening in some way. It is also possible that happy-averted gaze faces are particularly ambiguous in their intent, and it is this ambiguity that renders them interesting and increases our motivation to more elaborately encode details of the face when asked to actively commit them to WM. Work by Dugas et al. (2005) shows that individuals with high intolerance and worry for uncertain situations perceived ambiguous information to be more threatening and recalled more uncertainty-related than neutral words compared to individuals with lower intolerance levels for uncertainty. Individual differences were not measured in the current study, so no comment can be made in this regard to the pattern of results found here. Ambiguity in the context of averted gaze happy faces, however, might not necessarily be threatening but could signal an interesting and rewarding event in the environment such as the presence of something or someone considered to be pleasant (e.g., Bayliss, Schuch, \& Tipper, 2010). It would be fruitful for future research to explore in depth how perceived social intent is shaped 
by particular expression-gaze combinations, taking into account levels of anxiety and intolerance for uncertainty.

These findings add to growing evidence that expression and gaze information interact to alter perception of and memory for faces. An event-related potential (ERP) study showed that gaze and expression interact at $270-450 \mathrm{~ms}$ post-stimulus onset during a passive viewing task (Klucharev \& Sams, 2004), a time period thought to reflect stimulus evaluation and the analysis of meaning. What appears to be important, however, is that the resultant output of the analysis of expression-gaze combinations - the nature of intent conveyed - appears to rely on how and in what timeframe this information is used. When faces are explicitly judged on expression valence and intensity, certain expressions are perceived as less intense when gaze is averted, and vice versa, depending on the direction of intent (approach/avoid) (e.g., Adams \& Kleck, 2003, 2005), and there is evidence for an impact of gaze on judgements of a wide variety of expressions (Bindemann et al., 2008). But these effects do not map neatly onto expression-specific patterns in memory. Expression judgement tasks put perception and awareness of the emotion and what it might mean at the forefront of face processing, while gaze direction is somewhat incidental. In contrast, when memory for faces is measured both expression and gaze information is task-irrelevant, and despite this, certain combinations of these signals influence memory for face identity in memory specific-ways.

In addition to different encoding task demands as outlined above, one other difference exists between this WM study and Nakashima et al.'s LTM study which might be crucial. Nakashima and colleagues used Japanese participants and Japanese faces, while Caucasian participants and faces were used here. One study has shown that Japanese participants perceive faces with direct gaze as being angrier, less approachable, and more unpleasant compared to judgements made by Western (Finnish) Europeans (Akechi et al., 2013). Akechi and colleagues suggest that cultural norms and display rules can shape how we interpret certain expression-gaze combinations. More research is required to examine the effects of expression and gaze on faces which are more actively encoded into LTM, and to tease apart the potential added influence of culture on expression and gaze interpretation and its impact on LTM and WM. In addition, measures of clinical or subclinical depression and anxiety in future research of this nature may be useful, as these have been shown to influence WM performance (Joormann \& Gotlib, 2008; Linden, Jackson, Subramanian, Healy, \& Linden, 2011; Rose \& Ebmeier, 2006; Shackman et al., 2006), and can be prevalent in the undergraduate population often used for psychological research (Bayram \& Bilgel, 2008). 
In conclusion, effective interpretation and use of social cues which combine expression, gaze and identity information is a complex process which is not fully understood at present (see Graham \& LaBar, 2012). It seems that the ways in which gaze direction can alter expression and identity processing are context dependent and may be adaptive according to social, cognitive, and perhaps also cultural demands. When explicit and immediate processing of face identity into WM is engaged, averted gaze increases the potency of a smile, but the social intent conveyed by this expression-gaze combination remains a mystery. 


\section{References}

Adams, R.B. Jr. \& Kleck, R.E. (2003). Perceived gaze direction and the processing of facial displays of emotion. Psychological Science, 14, 644-647.

Adams, R.B. Jr. \& Kleck, R.E. (2005). Effects of directed and averted gaze on the perception of facially communicated emotion. Emotion, 5(1), 3-11.

Akechi, H., Senju, A., Uibo, H., Kikuchi, Y., Hasegawa, T., \& Hietanen, J.K. (2013).

Attention to eye contact in the West and East: Autonomic responses and evaluative ratings. PLoS ONE, 8(3): e59312.

Atkinson, R. C., \& Shiffrin, R. M. (1968). Human memory: A proposed system and its control processes. The Psychology of Learning \& Motivation, 2, 89-195.

Barton, J.J.S., Radcliffe, N., Cherkasova, M.V., Edelman, J., \& Intriligator, J.M. (2006). Information processing during face recognition: The effects of familiarity, inversion, and morphing on scanning fixations. Perception, 35(8), 1089-1105.

Bayliss, A.P., Schuch, S., \& Tipper, S.P. (2010). Gaze cuing elicited by emotional faces is influenced by affective context. Visual Cognition, 18(8), 1214-1232.

Bayram, N. \& Bilgel, N. (2008). The prevalence and socio-demographic correlations of depression, anxiety and stress among a group of university students. Social Psychiatry \& Psychiatric Epidemiology, 43(8), 667-672.

Bindemann, M., Burton, A.M., \& Langton, S.R.H. (2008). How do eye-gaze and facial expression interact? Visual Cognition, 16, 708-733.

D’Argembeau, A., \& Van der Linden, M. (2007). Facial expressions of emotion influence memory for facial identity in an automatic way. Emotion, 7(3), 507-515.

Dugas, M.J., Hedayati, M., Karavidas, A., Buhr, K., Francis, K., \& Phillips, N.A. (2005). Intolerance of uncertainty and information processing: Evidence of biased recall and interpretations. Cognitive Therapy \& Research, 29(1), 57-70.

Eisenbarth, H. \& Alpers, G.W. (2011). Happy mouth and sad eyes: Scanning emotional facial expressions. Emotion, 11(4), 860-865.s

Ekman, P. \& Friesen, W.V. (1976). Pictures of facial affect. Palo Alto, CA: Consulting Psychologists Press.

Farah, M.J., Wilson, K.D., Drain, H.M., \& Tanaka, J.N. (1995). The inverted face inversion effect in prosopagnosia: Evidence for mandatory, face-specific perceptual mechanisms. Vision Research, 35(14), 2089-2093. 
Freeth, M., Foulsham, T., \& Kingstone, A. (2013). What affects social attention? Social presence, eye contact, and autistic traits. PLoS ONE, 8(1): e53286.

Graham, R. \& LaBar, K.S. (2012). Neurocognitive mechanisms of gaze-expression interactions in face processing and social attention. Neuropsychologia, 50(5), 553-566.

Green, D.M. \& Swets, J.A. (1966). Signal Detection Theory and Psychophysics. New York: Wiley.

Jackson, M.C., Linden, D.E.J., \& Raymond, J.E. (2014). Angry expressions strengthen the encoding and maintenance of face identity representations in visual working memory. Cognition \& Emotion, 28(2), 278-297.

Jackson, M.C. \& Raymond, J.E. (2008). Familiarity enhances visual working memory for faces. Journal of Experimental Psychology: Human Perception \& Performance, 34(3), 556-568.

Jackson, M.C., Wolf, C., Johnston, S.J., Raymond, J.E., \& Linden, D.E.J. (2008). Neural correlates of enhanced visual short-term memory for angry faces: An fMRI study. PLoS ONE, 3(10), e3536.

Jackson, M.C., Wu, C-Y., Linden, D.E.J., \& Raymond, J.E. (2009). Enhanced visual shortterm memory for angry faces. Journal of Experimental Psychology: Human Perception \& Performance, 35(2), 363-374.

Joormann, J. \& Gotlib, I.H. (2008). Updating the contents of working memory in depression: Interference from irrelevant negative material. Journal of Abnormal Psychology, $117(1), 182-192$.

Kimble, C.E. \& Olszewski, D.A. (1980). Gaze and emotional expression: The effects of message positivity-negativity and emotional intensity. Journal of Research in Personality, 14(1), 60-69.

Kleinke, C.L. (1986). Gaze and eye contact: A research review. Psychological Bulletin, 100(1), 78-100.

Klucharev, V. \& Sams, M. (2004). Interaction of gaze direction and facial expressions processing: ERP study. Neuroreport, 15(4), 621-625.

Linden S.C., Jackson, M.C., Subramanian, L., Healy, D., Linden, D.E.J. (2011). Sad benefit in face working memory: An emotional bias of melancholic depression. Journal of Affective Disorders, 135, 251-257.

Liu, C.H., Chen, W., \& Ward, J. (2014). Remembering faces with emotional expressions. Frontiers in Psychology, 5, Article 1439. 
Mason, M., Hood, B., \& Macrae, C.N. (2004). Look into my eyes: Gaze direction and person memory. Memory, 12(5), 637-643.

Nakashima, S.F., Langton, S.R.H., \& Yoshikawa, S. (2012). The effect of facial expression ad gaze direction on memory for unfamiliar faces. Cognition \& Emotion, 26(7), 13161325 .

Nikolić, D. \& Singer, W. (2007). Creation of visual long-term memory. Perception \& Psychophysics, 69(6), 904-912.

Rose, E.J. \& Ebmeier, K.P. (2006). Pattern of impaired working memory during major depression. Journal of Affective Disorders, 90(2-3), 149-161.

Sander, D., Grandjean, D., Kaiser, S., Wehrle, T., \& Scherer, K.R. (2007). Interaction effects of perceived gaze direction and dynamic facial expression: Evidence for appraisal theories of emotion. European Journal of Cognitive Psychology, 19(3), 470-480.

Schurgin, M.W., Nelson, J., Iida, S., Ohira, H., Chiao, J.Y., \& Franconeri, S.L. (2014). Eye movements during emotion recognition in faces. Journal of Vision, 14(13):14, 1-16.

Schyns, P.G., Bonnar, L., \& Gosselin, F. (2002). Show me the features! Understanding recognition from the use of visual information. Psychological Science, 13(5), 402-409.

Sessa, P. \& Dalmaso, M. (2016). Race perception and gaze direction differently impair visual working memory for faces: An evet-related potential study. Social Neuroscience, 11(1), 97-107.

Sessa, P., Luria, R., Gotler, A., Jolicoeur, P., \& Dell'acqua, R. (2011). Interhemispheric ERP asymmetries over inferior parietal cortex reveal differential visual working memory maintenance for fearful versus neutral facial identities. Psychophysiology, 48(2), 187197.

Shackman, A.J., Sarinopoulos, I., Maxwell, J.S., Pizzagalli, D.A., Lavric, A., \& Davidson, R.J. (2006). Anxiety selectively disrupts visuospatial working memory. Emotion, 6(1), 40-61.

Shimamura, A.P., Ross, J.G., \& Bennett, H.D. (2006). Memory for facial expressions: The power of a smile. Psychonomic Bulletin \& Review, 13(2), 217-222.

Stiernströmer, E.S., Wolgast, M., \& Johansson, M. (2015). Effects of facial expression on working memory. International Journal of Psychology, 51(4), 312-317.

Thomas, P.M.J., Jackson, M.C., \& Raymond, J.E. (2014). A Threatening Face in the Crowd: Effects of Emotional Singletons on Visual Working Memory. Journal of Experimental Psychology: Human Perception \& Performance, 40(1), 253-263. 
Vrij, A. (2002). Deception in children: A literature review and implications for children's testimony. In H. L. Westcott, G. M. Davies, \& R. H. C. Bull (Eds.), Children's testimony (pp. 175-194). London: Wiley.

Young, S.G., Slepian, M.L., Wilson, J.P., \& Hugenberg, K. (2014). Averted eye-gaze disrupts holistic face encoding. Journal of Experimental Social Psychology, 53, 94-99. 